\title{
Otx2 expression is restricted to dopaminergic neurons of the ventral tegmental area in the adult brain
}

\author{
MICHELA DI SALVIO'ㄹ, LUCA G. DI GIOVANNANTONIO ${ }^{1}$, DANIELA OMODEI"1, \\ DARIO ACAMPORA ${ }^{1,3}$ and ANTONIO SIMEONE $*, 1,2,3$ \\ ${ }^{1}$ CEINGE Biotecnologie Avanzate, ${ }^{2}$ SEMM European School of Molecular Medicine and \\ 3 Institute of Genetics and Biophysics "A. Buzzati-Traverso", CNR, Naples, Italy
}

\begin{abstract}
Mesencephalic-diencephalic dopaminergic (mdDA) neurons control motor, sensorimotor and motivated behaviour and their degeneration or abnormal functioning is associated with important pathologies, such as Parkinson's disease and psychiatric disorders. Despite great efforts, the molecular basis and the genetic factors differentially controlling identity, survival and vulnerability to neurodegeneration of mdDA neurons of the substantia nigra (SN) and ventral tegmental area (VTA) are poorly understood. We have previously shown that the transcription factor Otx2 is required for identity, fate and proliferation of mesencephalic DA (mesDA) progenitors. By using mouse models and immunohistochemistry, we have investigated whether Otx 2 is expressed also in post-mitotic mdDA neurons. Our data reveal that Otx2 is expressed in postmitotic mesDA neurons during mid-late gestation and in the adult brain. Remarkably, Otx2 expression is sharply excluded from mdDA neurons of the $S N$ and is restricted to a relevant fraction of VTA neurons. Otx2 $2^{+}-\mathrm{TH}^{+}$neurons are concentrated to the ventral part of the VTA. Combined expression with other regionalized VTA markers shows that $0 \mathrm{tx} 2^{+}-\mathrm{TH}^{+}$neurons are prevalently Girk2- and $\mathrm{Calb}^{+}$and among these, those located in the medial and ventralmost portion of the VTA are also Ahd2 ${ }^{+}$. These findings indicate that Otx2 represents the first transcription factor with a proven role in mdDA neurogenesis whose expression discriminates between $\mathrm{SN}$ and a relevant proportion of VTA neurons. This supports the possibility that Otx 2 may act as a post-mitotic selector controlling functional features (e.g. identity and/or survival) of a relevant fraction of VTA neurons in the adult.
\end{abstract}

KEY WORDS: Otx2, mesencephalic-diencephalic, calbindin, Ahd2, Parkinson's disease

\section{Introduction}

Mesencephalic and diencephalic dopaminergic (mdDA) neurons are located in stereotypic positions corresponding to the ventral tegmental area (VTA), the substantia nigra (SN) and the retrorubral field (RRF) and originate from progenitors located in the floor plate region of the anterior prosomeres and ventral mesencephalon (Marìn et al., 2005; Smidt and Burbach, 2007; Hokfelt et al., 1984). The clinical relevance of mdDA neurons is high because of their regulatory and modulating functions in motor, sensorimotor and motivated behaviours (Jellinger, 2001). Degeneration of SN neurons leads to the characteristic symptoms of Parkinson's disease, while abnormal functioning of VTA neurons is involved in psychiatric disorders. These pathologies highlight the enormous effort to understand the molecular basis controlling generation, survival and functioning of mdDA neurons. Several gene functions including the transcription factors Pit 3 , Lmx1a, Lmx1b, En, Msx1, Foxa2, Ngn2 and Otx2 as well as the orphan nuclear receptor Nurr1, the Wnt1 and Wnt5a members of the Wnt family, the signalling molecules Shh and Fgf8 and the Retinoic Acid play a relevant role in specification, differentiation and survival of mdDA neurons (reviewed in Smidt and Burbach, 2007; Smits et al., 2006; Prakash and Wurst, 2006; Simeone,

\footnotetext{
Abbreviations used in this paper: Ahd2, aldehyde dehydrogenase family 1, subfamily A1 gene; Calb, calbindin D28K; Girk2, G-protein-gated inwardly rectifying $\mathrm{K}^{+}$channel subunit; mdDA neuron, mesencephalic-diencephalic dopaminergic neuron; mesDA progenitor, mesencephalic dopaminergic progenitor; SN, substantia nigra; TH, tyrosine hydroxylase; VTA, ventral tegmental area.
}

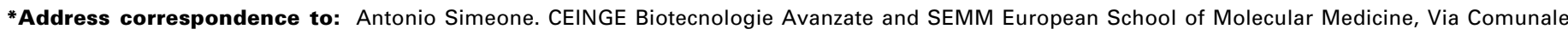
Margherita 482, 80145 Naples, Italy. Fax: +39-081-373-7808. e-mail: simeone@ceinge.unina.it 
2005). However, despite the great efforts, very little is known about the mechanism controlling post-mitotic identity and functional features of VTA and SN neurons. For example and strictly related to Parkinson's disease, the molecular basis determining for SN neurons a higher sensitivity to neurodegeneration as compared to VTA neurons is virtually unknown. Interestingly, the fact that several gene-markers have been reported to be expressed in specific subsets of VTA and SN neurons (Chung et al., 2005; Greene et al., 2005), suggests that the VTA and SN may include multiple neuronal subpopulations, whose functional features may be different. In this context, Calbindin D28K (Calb) and Girk2 are believed to be respectively expressed in VTA neurons more resistant to and $\mathrm{SN}$ neurons more vulnerable to neurodegeneration; similarly, the aldehyde dehydrogenase family 1, subfamily A1 gene, also known as Ahd2, or Aldh1a1 or Raldh1, is expressed in the SN and in a ventral subset of VTA neurons and is transcriptionally controlled by Pitx3, which, although ubiquitary expressed in mdDA neurons, is apparently required only for the development of SN neurons (Smidt and Burbach, 2007; Murer et al., 1997; Liang et al.,1996; McCaffery and Drager, 1994; Schein et al., 1998; Jacobs et al., 2007). These findings reinforce the idea that VTA and SN neurons require further genetic instructions regulating maintenance, survival, natural cell-death and sensitivity to degeneration during postmitotic maturation and adult life. We previously concentrated our efforts on the transcription factor Otx2, which is required for the establishment of the molecular code of progenitor domains in the

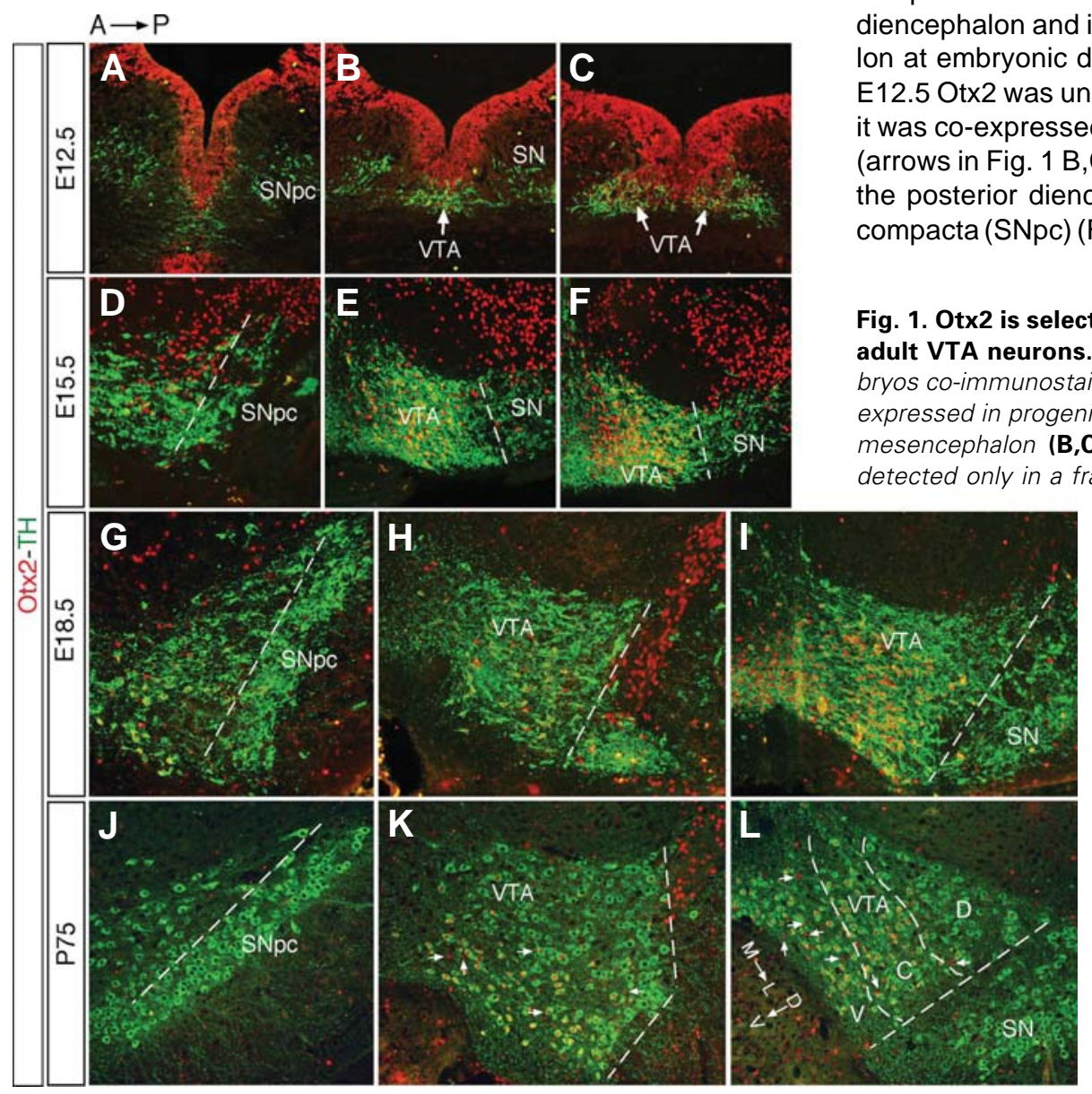

ventral mesencephalon and, as an intrinsic factor, for the neurogenesis of mesencephalic DA (mesDA) neurons (Puelles et al., 2003; 2004; Prakash et al., 2006; Omodei et al., 2008). Based on this, we hypothesized that, if expressed in post-mitotic and/or adult mdDA neurons, Otx2 might play additional roles in these neurons. We discovered that Otx2 is expressed selectively in post-mitotic mesDA neurons during embryonic development and adult life. Importantly, its expression is restricted to a relevant percentage of VTA neurons and excluded from those of the SN. Most of the Otx2+ neurons are Girk2- and $\mathrm{Calb}^{+}$and those located in the medial and ventralmost part of the VTA are also Ahd2+. These data support the possibility that Otx2 may be required in embryos and/or adult mice as a post-mitotic selector controlling functional features (eg. identity and/or survival) of VTA neurons.

\section{Results}

\section{Otx2 is expressed in developing post-mitotic mesDA neu- rons and is restricted to VTA neurons in the adult}

Previous studies have indicated that Otx2 is expressed in the mdDA progenitor domain along the mesencephalon and posterior diencephalon, and that it is required to control selectively differentiation and proliferation of mesDA progenitors (Omodei et al., 2008). We now revisited in detail the expression profile of Otx2 during mid-late development and in the adult brain to assess whether Otx2 was also expressed in embryonic post-mitotic and adult mdDA neurons. To this aim, the expression of Otx2 was first compared to that of the Tyrosine Hydroxylase $(\mathrm{TH})$ in the posterior diencephalon and in the anterior and intermediate mesencephaIon at embryonic day (E) 12.5, E15.5 and E18.5 (Fig. 1 A-I). At E12.5 Otx2 was uniformly expressed in mdDA progenitors, while it was co-expressed with $\mathrm{TH}$ only in a fraction of mesDA neurons (arrows in Fig. $1 \mathrm{~B}, \mathrm{C}$ ) and was excluded from those originating in the posterior diencephalon and fated to generate the SN pars compacta (SNpc) (Fig. 1A). The restricted expression of Otx2 was

Fig. 1. Otx2 is selectively expressed in embryonic post-mitotic and adult VTA neurons. (A-C) Adjacent transverse sections of E12.5 embryos co-immunostained with Otx2 and TH show that Otx2 is uniformly expressed in progenitor domains of the posterior diencephalon (A) and mesencephalon (B,C), while in post-mitotic $\mathrm{TH}^{+}$mesDA neurons is detected only in a fraction of them (arrows in B,C) and excluded from those of diencephalic origin fated to generate primarily the SNpc (A). (D-I) At E15.5 and E18.5, Otx2 ${ }^{+}-\mathrm{TH}^{+}$neurons are absent from the SNpc (D,G), while in the VTA exhibit graded A-P numerical increase $(\mathbf{E}, \mathbf{F}, \mathbf{H}, \mathbf{I})$. (J-L) At P75 the expression of Otx2 is maintained in $\mathrm{TH}^{+}$neurons located in the ventral and central VTA $(\mathbf{K}, \mathbf{L})$, and absent from those of the SN (J). Dotted lines demarcate the presumptive boundary between SN and VTA at E12.5 and E15.5, and the definitive boundary at E18.5 and P75. In (L) the VTA is subdivided arbitrarily in dorsal, central and ventral. The arrows in $(\mathbf{K}, \mathbf{L})$ point to sporadic Otx2 $2^{+}-\mathrm{TH}^{-}$cells. Abbreviations: $S N$, substantia nigra; $S N p c$, substantia nigra pars compacta; VTA, ventral tegmental area; $A \rightarrow P$, anterior $\rightarrow$ posterior; $D, C, V$ stand for dorsal, central and ventral VTA, respectively; $M \rightarrow L$ and $D \rightarrow V$ stand for medial $\rightarrow$ lateral and dorsal $\rightarrow$ ventral. 
more evident at E15.5 and E18.5. At these stages, Otx2 was absent in the diencephalic and mesencephalic SNpc neurons (Fig. $1 \mathrm{D}, \mathrm{G}$ ), and expressed in a relevant number of VTA neurons (Fig. $1 \mathrm{E}, \mathrm{F}, \mathrm{H}, \mathrm{I})$. In the adult brain at around post-natal day 75 (P75), the expression of Otx2 was maintained selectively in $\mathrm{TH}^{+}$ neurons of the VTA and absent in those of the SN (Fig. $1 \mathrm{~J}-\mathrm{L}$ ). In particular, the Otx2 ${ }^{+}-\mathrm{TH}^{+}$neurons appeared prevalently confined to the central and ventral VTA (Fig. $1 \mathrm{~K}, \mathrm{~L})$. Detailed analysis along the anterior-posterior $(A-P)$ axis of the mesencephalon and cell-counting of $\mathrm{Otx}^{+}-\mathrm{TH}^{+}$neurons over the total number of $\mathrm{VTA} \mathrm{TH}^{+}$neurons, showed that in the anterior VTA (Fig. 1K) $28 \%$ of $\mathrm{TH}^{+}$neurons were Otx2 $2^{+}$and in the intermediate VTA (Fig. 1L), this percentage was increased up to $65 \%$. Moreover, the density of Otx2 $2^{+}-\mathrm{TH}^{+}$neurons showed a graded distribution also along the dorsal-ventral axis of the VTA. To evaluate numerically this distribution we arbitrarily subdivided the VTA of the intermediate mesencephaIon in three areas: dorsal, central and ventral (Fig. 1L) and determined the percentage of $\mathrm{Otx} 2^{+}-\mathrm{TH}^{+}$neurons in these areas. In the dorsal, central and ventral domains the percentage of $\mathrm{TH}^{+}$neurons exhibiting Otx2 expression was $8 \%, 37 \%$ and $75 \%$, respectively. However, in this analysis we also identified a small percentage $(7 \%)$ of Otx2 $2^{+} \mathrm{TH}^{-}$neurons located within the VTA (arrows in Fig. $1 \mathrm{~K}, \mathrm{~L}$ ). Thus, these findings indicate that Otx2 represents a molecular correlate of a restricted population of post-mitotic and adult mesDA neurons prevalently distributed in the ventral and central VTA. This supports the possibility that Otx2 may also be required as a selector controlling functional features of a restricted population of embryonic post-mitotic and/ or adult VTA neurons. Then, since the Otx2 antibody recognizes, although at a reduced efficiency, also Otx1, we analyzed whether the expression profile previously shown was due to Otx2 or Otx1 or to both. To this aim we first analyzed a mouse model in which the Otx2 coding sequence and introns were replaced by the GFP reporter gene (Acampora et al., 2009). In E18.5 and adult Otx $2^{G F P /+}$ mice, the GFP expression sharply colocalized with TH and Otx2 in VTA neurons (Fig. 2 D-O), while no $\mathrm{TH}^{+}-\mathrm{GFP}^{+}$neurons were detected in the SN (Fig. $2 \mathrm{~A}-\mathrm{C}$ ), thus confirming that the staining previously shown was due to Otx2 expression. To assess the possibility that also Otx 1 was expressed in these neurons, we analyzed Otx1 null mutants carrying the Cre activity under the control of Otx1

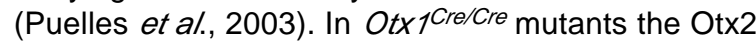
staining was unaffected (Fig. $3 \mathrm{~A}, \mathrm{~B}$ ) and no $\mathrm{TH}^{+}-\mathrm{Cre}^{+}$ neurons were identified (Fig. $3 C, D$ ), thus ruling out the possibility that also Otx1 was expressed in mdDA neurons. Similar results were observed also in Otx $1 \mathrm{Cre} / \mathrm{Cre}$ embryos (data not shown).

\section{Otx2 expression defines different subsets of VTA DA neurons}

To determine whether the Otx2 expression may be correlated to specific subsets of adult VTA neurons, we first analyzed at single cell level the expression of several VTA neuronal markers and, subsequently, correlated the Otx2 expression to them. At E18.5, we analyzed the expression of Pitx3, Ahd2 and Calb and in adult brains also that of Girk2, which was not expressed before P10. Pitx3 was co-expressed with $\mathrm{TH}$ even tough its expression level appeared reproducibly not uniform in all neurons and in some of them was undetectable (Fig. 4 A,E,J). As previously reported (McCaffery and Drager,

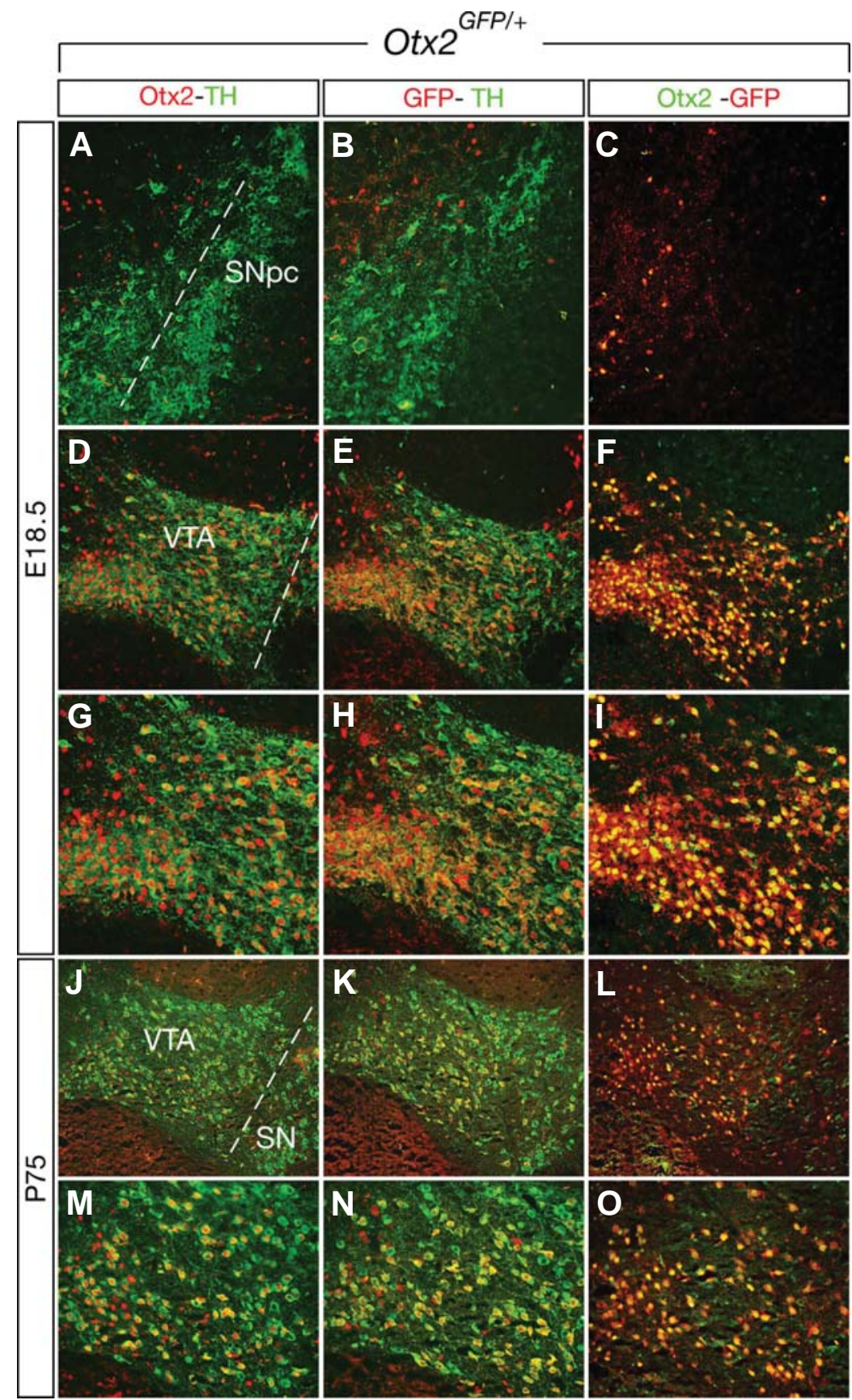

Fig. 2. Otx2-driven GFP expression confirms Otx2 expression in $\mathrm{TH}^{+}$mesDA neurons. (A-O) Transverse adjacent sections of E18.5 (A-I) and P75 (J-O) Otx2 ${ }^{\mathrm{GFP} /+}$ mutants immunostained with Otx2 and TH, GFP and TH, and Otx2 and GFP show that Otx2 and GFP sharply colocalize in $\mathrm{TH}^{+}$neurons of the VTA (D-O) and are absent in those of the $S N(\mathbf{A}-\mathbf{C})$. (G-I) and (M-O) are magnification of (D-F) and (J-L) respectively. Abbreviations as in Fig. 1. 


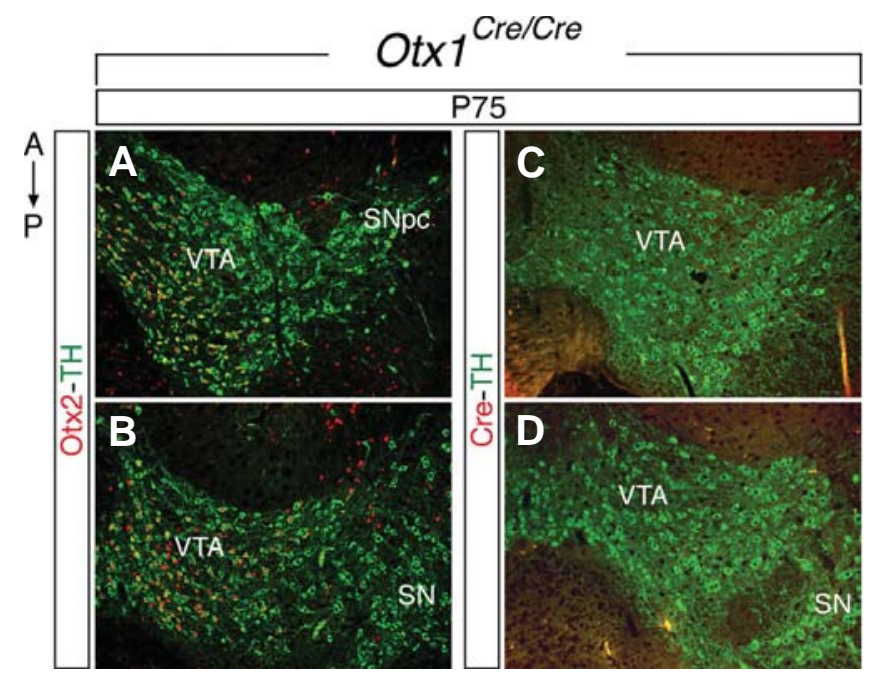

Fig. 3. Otx1 is not expressed in VTA neurons. (A-D) Transverse sections of a P75 Otx 1 Cre/Cre mouse immunostained with Otx2 and TH $(\mathbf{A}, \mathbf{B})$ and $C r e$ and $\mathrm{TH}(\mathbf{C}, \mathbf{D})$ show no variation in the number of $\mathrm{Ot} \times 2^{+}-\mathrm{TH}^{+}$ neurons and no staining for Otx1-driven Cre activity. Abbreviations as in Fig. 1.

1994; Jacobs et al., 2007), Ahd2 was prevalently expressed in $\mathrm{TH}^{+}$neurons located in the medialventral part of the VTA and in some scattered neurons distributed in the central VTA (Fig. 4 $B, F, K)$. Calb was expressed at E18.5 in a relatively low percentage of neurons distributed all along the dorsal-ventral (D-V) axis of the VTA (Fig. 4C). Calb+- $\mathrm{TH}^{+}$neurons were scarcely detected at this stage in the medial part of the VTA, where the Ahd2 expression was predominant (arrow in Fig. 4 B-D). In the adult brain, Calb was instead expressed in most of the $\mathrm{TH}^{+}$neurons with the exception of those concentrated in the lateral-dorsal corner of the VTA (Fig. 4 G,L).

Fig. 4. Marker analysis highlights different subpopulations of $\mathrm{TH}^{+}$neurons in the VTA. (A-D) Transverse adjacent sections at E18.5 immunostained with Pitx 3 and TH(A), Ahd 2 and TH(B), Calb and TH(C) and Calb and Ahd2 (D) show that Pitx 3 is expressed at high level in most but not in all of the VTA TH+ neurons (A); Ahd 2 is prevalently expressed in the medial and ventral VTA (arrow in B); Calb is detected in the central and lateral VTA all along the dorsal-ventral axis and excluded medially (arrow in C), as it is evident in Ahd2-Calb coimmunostaining (arrow in D). (E-N) Transverse adjacent sections of a P75 adult brain immunostained with Pitx3 and $T H(\mathbf{E}, \mathbf{J})$, Ahd 2 and $T H(\mathbf{F}, \mathbf{K})$, Calb and $T H(\mathbf{G}, \mathbf{L})$, Calb and $A$ hd $2(\mathbf{H}, \mathbf{M})$ and Girk2 and $T H(\mathbf{I}, \mathbf{N})$ show that even in the adult brain Pitx 3 is expressed at high level in most but not in all of the $\mathrm{TH}^{+}$neurons $(\mathbf{E}, \mathbf{J})$; Ahd 2 is restricted to $\mathrm{TH}^{+}$neurons located along the ventral and medial VTA (F,K); Calb is expressed in most of the $\mathrm{TH}^{+}$neurons $(\mathbf{G}, \mathbf{L})$ including those located medially and expressing Ahd $2(\mathbf{H}, \mathbf{M})$, while it is detected in a reduced number of $\mathrm{TH}^{+}$ neurons located in the lateral-dorsal corner of the VTA where Girk2 is aboundantly detected $\mathbf{( I , N )}$. The dotted line in (E) demarcates the SN and VTA; the dotted lines in (F-I) demarcate the dorsal, central and ventral VTA; the square in (E) demarcates the area shown in (J-N). Abbreviations as in Fig. 1.
Noteworthy and compared to E18.5 embryos, in the adult VTA most if not all the Ahd2+ neurons were Calb+ (Fig. $4 \mathrm{H}, \mathrm{M}$ ), thus suggesting further refinement of $\mathrm{TH}^{+}$neurons in their post-mitotic identity during post-natal life. Finally, we analyzed the expression of Girk2 (Schein et al., 1998; Andersson et al., 2006), which was expressed at high level prevalently in the dorsal-lateral part of the VTA, where it seemed to mirror the Ahd2 expression profile in the medial-ventral part of the VTA (Fig. 4 I,N). Thus, through this expression analysis and previous studies (McCaffery and Drager, 1994; Smidt et al., 1997; Murer et al., 1997; Jacobs et al., 2007; Liang et al., 1996), we have defined several $\mathrm{TH}^{+}$subpopulations of adult VTA neurons. In particular, the majority of $\mathrm{TH}^{+}$neurons were $\mathrm{Calb}^{+}$, and, among these, those in the medial and ventral VTA co-expressed also Ahd2; conversely, $\mathrm{TH}^{+}$neurons located in lateral-dorsal corner of the VTA exhibited strong expression of Girk2 and a fraction of them were Calb- Based on this expression profile, we analyzed the expression of Otx2 to provide further information on its potential role in specific VTA neuronal subpopulation(s). As previously shown (Fig. 1), almost all (93\%) of the Otx2 ${ }^{+}$neurons were $\mathrm{TH}^{+}$and were concentrated prevalently in the ventral and central VTA (Fig. 5 A,E). Comparison with Pitx3 revealed that most of the Otx2+ neurons were Pitx3 ${ }^{+}$(Fig. 5 B,F). However, we noticed that about $20 \%(22 \%)$ of the Otx $2^{+}$neurons

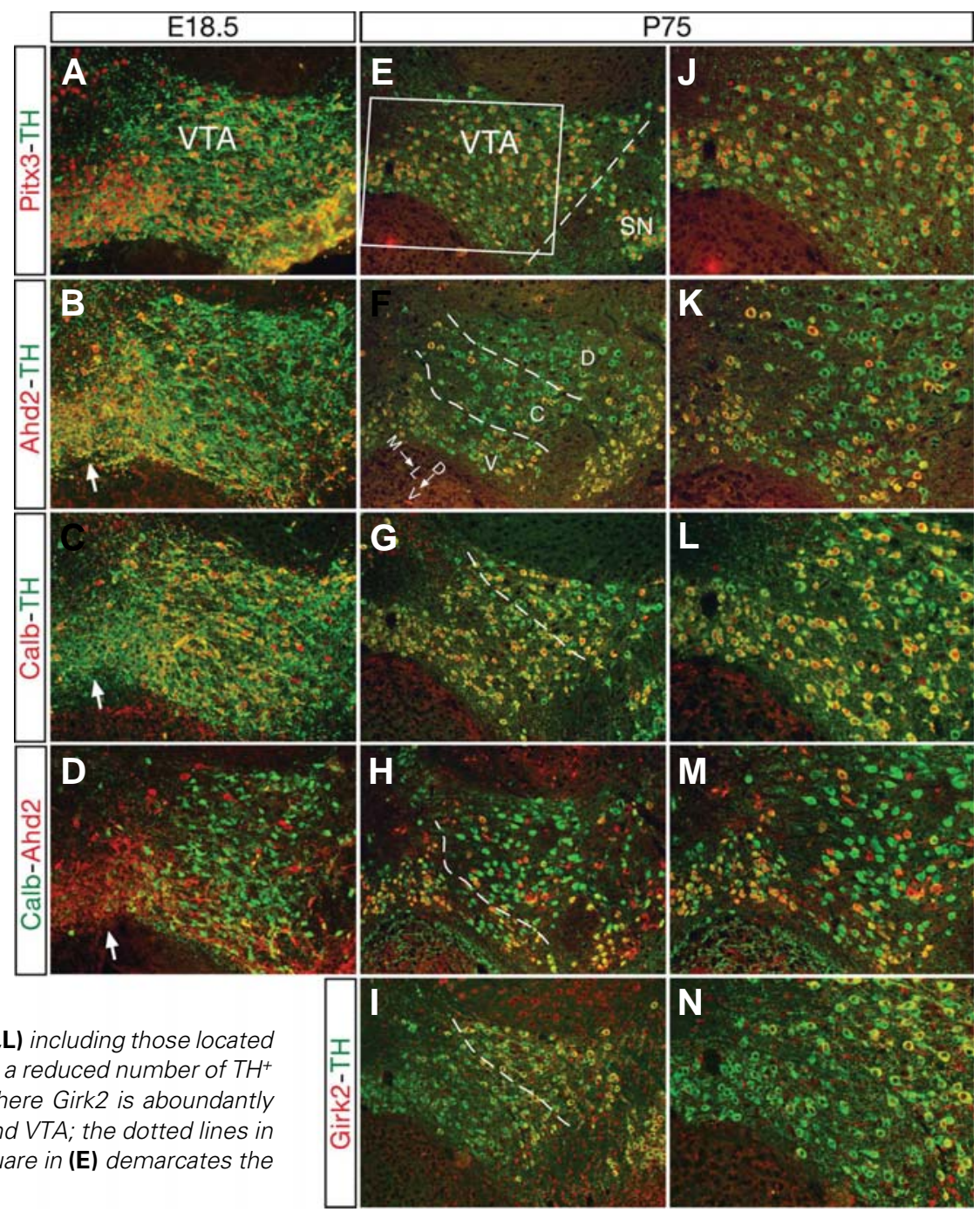


exhibited weak or almost undetectable expression of Pitx3 (Fig. $5 \mathrm{~B}, \mathrm{~F})$. At E18.5 the majority of Otx2 ${ }^{+}$neurons in the medial VTA were Calb $^{-}$and $\mathrm{And}^{+}$(arrow in Fig. 5 C,D) while, among those located more dorsally in the central VTA, a relevant fraction did express Calb (Fig. 5C). In the adult the expression profile was different. Indeed, in the central VTA most of the Otx2 ${ }^{+}$neurons were Ahd2-Calb+ (arrows Fig. $5 \mathrm{G}, \mathrm{H}$ ), while in the ventral VTA, Otx2 ${ }^{+}$neurons were in the large majority $\mathrm{Calb}^{+}$-Ahd2 ${ }^{+}$(Fig. 5 $\mathrm{G}, \mathrm{H})$. Finally, comparison with Girk2 showed that Girk2 and Otx2 were only sporadically co-expressed in the dorsal VTA (arrows in Fig. 5I). However, since Girk2 was expressed at a low level also in the rest of the VTA, a few Otx2 ${ }^{+}$-Girk2 ${ }^{+}$neurons could be found in the central and ventral VTA (Fig. 5I). This analysis indicates that Otx2 is sporadically expressed in $\mathrm{TH}^{+}$neurons of the dorsal VTA where Girk2 is aboundant, while it is prevalently detected together with Calb in a population of $\mathrm{TH}^{+}$neurons located in the central VTA, and with Ahd2 and Calb in $\mathrm{TH}^{+}$neurons of the ventral VTA (Fig. 6).

\section{Discussion}

Previous studies indicated that: i) Otx2 together with Otx1 is required to control the positioning of Shhand Fgfoexpression and that failure in this control generates profound alteration in the identity code of progenitor domains in the ventral mesencephalon (Puelles et al., 2003); ii) Otx2 is required in the ventral mesencephalon to suppress the transcription factor Nkx2.2 and prevents the generation of Serotoninergic neurons in place of red nucleus and dorsalmost mesDA neurons (Puelles et al., 2004); iii) Wnt1 and Otx2 may be engaged in a positive feedback loop, which, is required for proper development of mesDA neurons (Prakash et al., 2006); and iv) Otx2 is intrinsically required to control mesDA neurogenesis through a graded A-P effect on the proliferation and differentiation of mesDA progenitors (Omodei et al., 2008). On the basis of these findings we have hypothesized that if expressed in post-mitotic embryonic and/or adult mdDA neurons, Otx2 might be involved also in controlling post-mitotic aspects of mdDA neuronal differention and, in the adult brain in proper functioning of mdDA neurons. In a first attempt, we have analyzed in detail the Otx2 expression during the post-mitotic transition of mdDA progenitors and their maturation during midlate development, and then, whether Otx2 is a molecular correlate of adult mdDA neurons. At E12.5 Otx2 is aboundantly expressed in mdDA progenitors located in the floor plate region of the posterior diencephalon and mesencephalon, while in differenti-

Fig. 5. Otx2 expression is prevalently associated with $\mathrm{TH}^{+}$neurons expressing Calb or both Calb and Ahd2. (A-D) Transverse adjacent sections at E18.5 immunostained with Otx2 and TH(A), Otx2 and Pitx3 (B), Otx2 and Calb (C) and Otx2 and Ahd2 (D) show that most of the Otx2 $2^{+}$neurons are $\mathrm{TH}^{+}(\mathbf{A})$ and Pit $3^{+}(\mathbf{B})$; those located in the medial VTA are prevalently Ahd $2^{+}$(arrow in D) and only a fraction of them are at this stage Calb+ (arrow in C). (E-I) Transverse adjacent sections of a P75 adult brain immunostained with Otx2 and TH(E), Otx2 and Pitx3 (F), Otx2 and Calb (G), Otx2 and Ahd2 (H), and Otx2 and Girk2 (I) show that most of the $\mathrm{Otx}^{+}$cells are $\mathrm{TH}^{+}(\mathbf{E})$; about $75 \%$ of them exhibit high leve of Pitx3 (F); most of them are Calb+ $(\mathbf{G})$; and those located in the medial and ventral VTA are also Ahd $2^{+}(\mathbf{H})$; while, in contrast, only sporadically Ot $\times 2^{+}$neurons are Girk2+ (arrows in I). The arrows in (E) point to rare

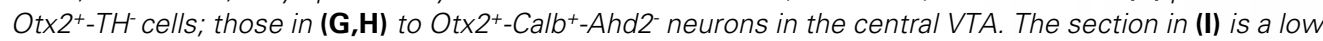
magnification to better show the complementary expression of Otx2 and Girk2. The dotted lines in (E,GI) demarcate the dorsal, central and ventral VTA. Abbreviations as in Fig. 1 ated $\mathrm{TH}^{+}$neurons is detected only in a fraction of those originating from mesDA progenitors and fated to populate the VTA. Detailed inspection of embryos and cell-counting of Otx2 $2^{+}-\mathrm{TH}^{+}$neurons in the adult VTA, showed that their percentage gradually increases along the A-P axis. This finding correlates with a similar polarity previously reported for Otx2 in the control of proliferation and differentiation of mesDA progenitors (Omodei et al., 2008) and suggests the existence of a fine mechanism preventing Otx2 expression in $\mathrm{TH}^{+}$neurons fated to generate the SN. We speculate that this mechanism should operate prevalently during postmitotic transition of progenitors fated to generate SN neurons and should be extremely efficient, since essentially no adult $\mathrm{SN}$ cells express Otx2. This study also shows that the restricted expression of Otx2 represents a stable molecular correlate restricted to the VTA even during post-natal life at least until one year of age (data not shown). The observation that Otx2 is selectively ex-

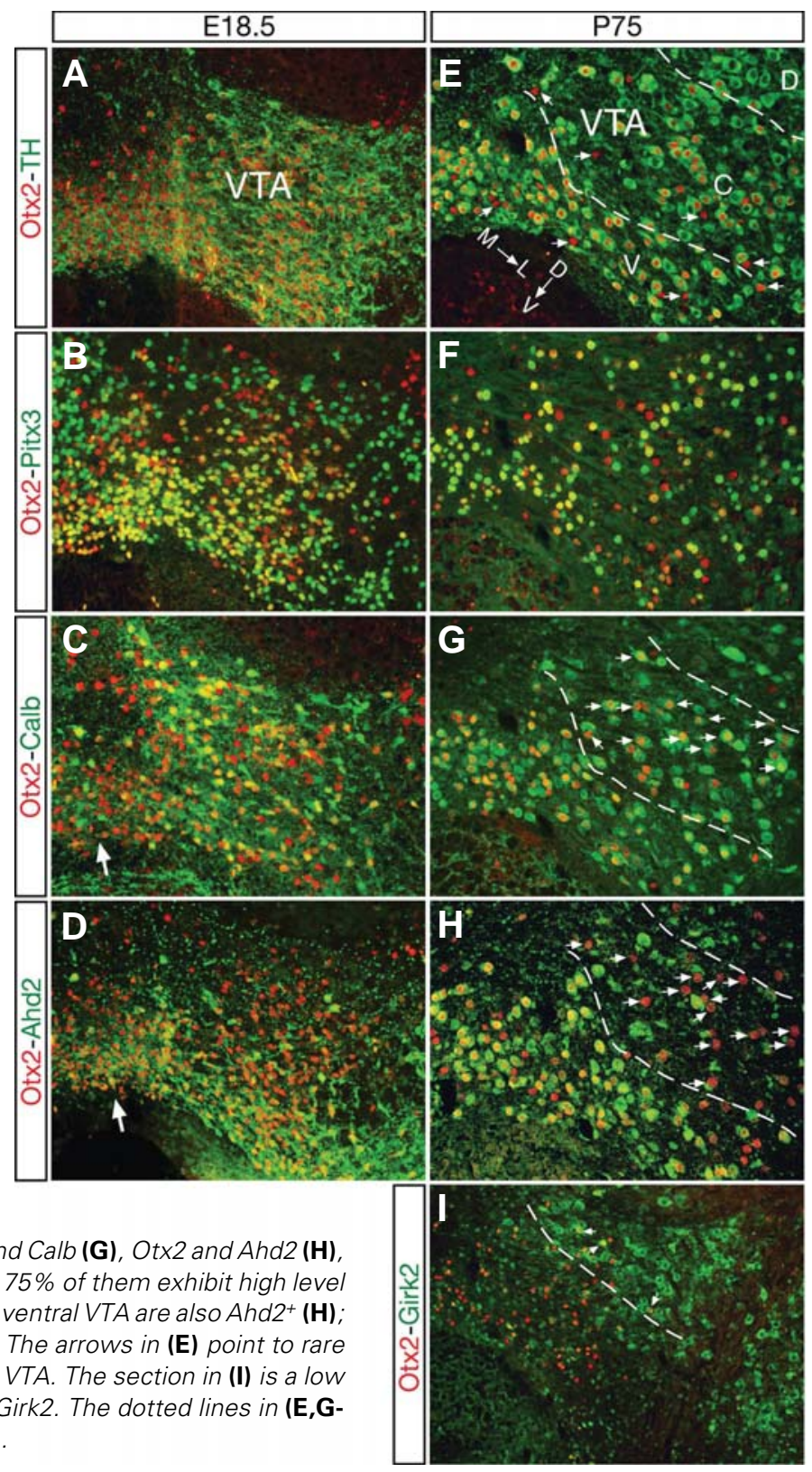


pressed in a relevant fraction of VTA neurons and absent in SN neurons represents, in our opinion, the major finding of this study. Indeed, despite the great effort lavished to identify selective markers and genetic functions required to maintain and/or specify identity, survival and differential vulnerability to neurodegeneration of the SN and VTA neurons, very little is known about these topics, and so far, very few, if any, gene functions have been shown to be exclusively expressed in the VTA or SN. Nevertheless, several genes (eg. Girk2, Ahd2, Calb) are preferentially but not exclusively expressed in SN or VTA neurons (Liang et al., 1996; Jacobs et al., 2007; Greene et al., 2005; Chung et al., 2005; Wallén et al., 1999; Schein et al., 1998). Alternatively, gene functions such as Pitx3, Nurr1, Lmx1b, En1 and Foxa2, which play a crucial role in specification and survival of mdDA neurons, are ubiquitously expressed in post-mitotic embryonic and adult VTA/SN neurons (reviewed in Smidt and Burbach, 2007; Smits etal., 2006; Sonnier et al., 2007; Prakash and Wurst, 2006; Simeone, 2005). In this context, Otx2 represents, to our knowledge, the first transcription factor with a crucial role in mesDA neurogenesis that is exclusively expressed in VTA neurons, being sharply excluded from those of the SN. A last finding of this study is the observation that, although restricted to VTA neurons, Otx2 is expressed only in a subset of them thus reinforcing the idea that VTA neurons are differentially patterned and, on the basis of their expression code and position, may represent neuronal subpopulations with different functional properties. This possibility is also supported by the restricted expression exhibited by Calb, Girk2 and Ahd2, and the phenotypic analysis of Pitx3 mutants. Indeed Calb, Girk2 and Ahd2 are expressed in complementary and/or overlapping subpopulations of VTA neurons (Liang et al., 1996; Schein et al., 1998; McCaffery and Drager, 1994) and lack of Pitx3 which is

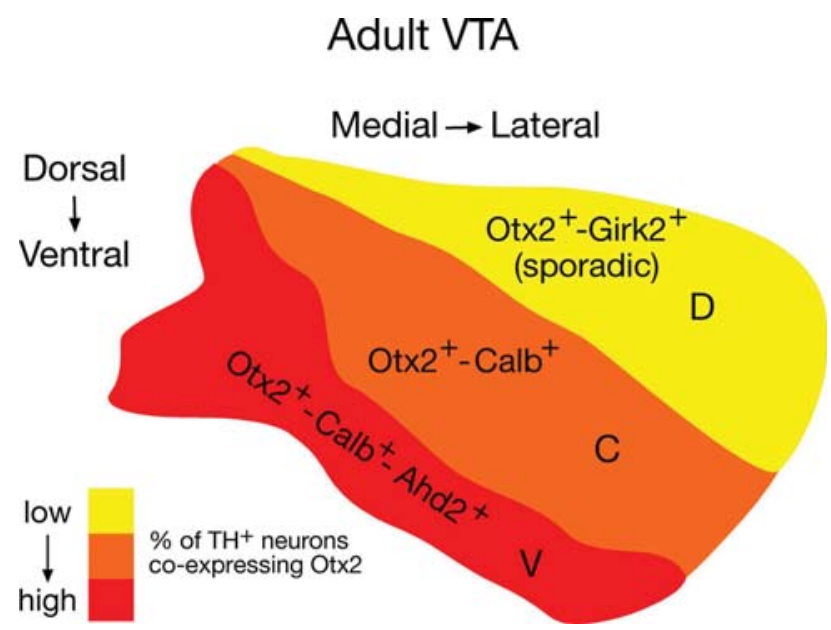

Fig. 6. Schematic representation summarizing Otx2 expression in the VTA of adult mice. Otx2 is prevalently detected in $\mathrm{TH}^{+}$neurons located in the central and ventral VTA with a graded increase along the dorsal-ventral axis. In particular, cell counting analysis indicates that in the central VTA (orange) $37 \%$ of the $\mathrm{TH}^{+}$neurons are Otx2+, in the ventral VTA (red) $75 \%$ of the $\mathrm{TH}^{+}$neurons are Otx2+; while in the dorsal VTA less then $10 \%$ of the $\mathrm{TH}^{+}$neurons are Otx2+. Our data also show that while Otx2 is sporadically co-expressed with Girk2, most if not all of the Otx2+ neurons are $\mathrm{Calb}^{+}$, and those located in the ventral VTA are also Ahd2+. Abbreviations as in Fig. 1. expressed in virtually all mdDa neurons generates the selective loss of SN neurons (reviewed in Smidt and Burbach 2007; Simeone 2005). Otx2 expression is almost completely absent in the dorsal-lateral corner of the VTA where $\mathrm{TH}^{+}$neurons heavily co-expressed Girk2; while it is co-expressed with Calb in the central VTA and with both Calb and Ahd 2 in the medial and ventral VTA (Fig. 6). Noteworthy, the percentage of $\mathrm{TH}^{+}$neurons coexpressing Otx2 exhibits a graded increase along the D-V axis of the VTA ( $8 \%, 37 \%$ and $75 \%$ in the dorsal, central and ventral VTA, respectively) (Fig. 6). This finding suggests that VTA neurons may be regionalized in subpopulations of differentially specified neurons. In this context it has been reported that Calb and Girk2 are respectively expressed in VTA neurons more resistant to and SN neurons more vulnerable to neurodegeneration (Chung et al., 2005; Greene et al., 2005; Liang et al., 1996; Roffler-Tarlov et al., 1996). Whether Otx2 is required to confer functional features determining identity, survival or resistance to neurodegeneration of VTA neurons, is now an attracting possibility to be investigated in the near future.

\section{Materials and Methods}

\section{Immunohistochemistry}

Immunohistochemistry experiments were performed as described (Omodei et al., 2008). The rabbit antibodies were directed against Otx2 (Omodei et al., 2008), Pitx3 (Zymed-Invitrogen), Ahd2 (Abcam), Calb (Swant), Girk2 (Alomone Labs), Cre (Novagen), and GFP (MBL); the mouse antibody against $\mathrm{TH}$ (Chemicon); and the goat antibodies against Otx2 (R\&D Systems), Pitx3 (Santa Cruz), Calb (R\&D System) and Ahd2 (Abcam)

\section{Dopaminergic cell counting}

Four adult brains (P75) (B6D2 strain) were sectioned in 5 series and one series was immunostained with $\mathrm{TH}$. Three sequential sections similar to those shown in (Fig. $1 \mathrm{~K}, \mathrm{~L})$, were selected at each of the two anatomical levels corresponding to the anterior and intermediate mesencephalon. Sections were immunostained with Otx2 and TH, Otx2 and Calb, Otx2 and Ahd2, and Otx2 and Girk2. Immunostained sections were photographed and each section printed in A4 format to allow a more precise cell counting. The percentage of $\mathrm{TH}^{+}$VTA neurons expressing Otx2 as well as the percentage of Otx2 $2^{+}$neurons co-expressing Calb or Ahd2, or Girk2, was determined. No relevant variability in cell number was observed among the different brains analyzed.

\section{Acknowledgements}

This work was supported by the FP7 for the project mdDANEURODEV (222999), the Italian Association for Cancer Research (AIRC), the FP6 for the EUTRACC Integrate Project (LSHG-CT-2007-037445), the "Stem Cell Project" of Fondazione Roma and the MIUR for the PRIN project (20079ZLWYP_003).

\section{References}

ACAMPORA, D., DI GIOVANNANTONIO, L.G., DI SALVIO, M., MANCUSO P. and SIMEONE A. Selective inactivation of Otx2 mRNA isoforms reveals isoformspecific requirement for visceral endoderm anteriorization and head morphogenesis and highlights cell diversity in the visceral endoderm. Mech Dev 126: 882-897.

ANDERSSON, E., JENSEN, J.B., PARMAR, M., GUILLEMOT, F. and BJÖRKLUND, A. (2006). Development of the mesencephalic dopaminergic neuron system is compromised in the absence of neurogenin 2. Development 133: 507-516.

CHUNG, C.Y., SEO, H., SONNTAG, K.C., BROOKS, A., LIN, L. and ISACSON, O. (2005). Cell type-specific gene expression of midbrain dopaminergic neurons 
reveals molecules involved in their vulnerability and protection. Hum Mol Genet 14: 1709-1725.

GREENE, J.G., DINGLEDINE, R. and GREENAMYRE, J.T. (2005). Gene expression profiling of rat midbrain dopamine neurons: implications for selective vulnerability in parkinsonism. Neurobio/ Dis 18: 19-31

HÖKFELT, T., MATENSSON, A., BJÖRKLUND, S., KLEINAU, S. and GOLDSTEIN, M. (1984). Distributional maps of tyrosine hydroxylase-immunoreactive neurons in the rat brain. In Handbook of Chemical Neuroanatomy: Classical Transmitters in the CNS(Eds. A. Björklund and T. Hökfelt), Elsevier, Amsterdam, 2: $227-379$

JACOBS, F.M., SMITS, S.M., NOORLANDER, C,W., VON OERTHEL, L., VAN DER LINDEN, A.J., BURBACH, J.P. and SMIDT, M.P. (2007). Retinoic acid counteracts developmental defects in the substantia nigra caused by Pitx 3 deficiency. Development 134:2673-2684.

JELLINGER, K.A. (2001). The pathology of Parkinson's disease. AdvNeuro/86: 55 72.

LIANG, C.L., SINTON, C.M., SONSALLA, P.K. and GERMAN, D.C. (1996). Midbrain dopaminergic neurons in the mouse that contain calbindin-D28k exhibit reduced vulnerability to MPTP-induced neurodegeneration. Neurodegeneration 5: 313-318

MARÍN, F., HERRERO, M.T., VYAS, S. and PUELLES, L. (2005). Ontogeny of tyrosine hydroxylase mRNA expression in mid- and forebrain: neuromeric pattern and novel positive regions. Dev Dyn 234: 709-717.

McCAFFERY, P. and DRÄGER, U.C. (1994). High levels of a retinoic acidgenerating dehydrogenase in the meso-telencephalic dopamine system. Proc Natl Acad Sci USA 91: 7772-7776.

MURER, G., ADELBRECHT, C., LAURITZEN, I., LESAGE, F., LAZDUNSKI, M. AGID, Y. and RAISMAN-VOZARI, R. (1997). An immunocytochemical study on the distribution of two G-protein-gated inward rectifier potassium channels (GIRK2 and GIRK4) in the adult rat brain. Neuroscience 8: 345-357.

OMODEI, D., ACAMPORA, D., MANCUSO, P., PRAKASH, N., DI GIOVANNANTONIO, L.G., WURST, W. and SIMEONE, A. (2008). Anteriorposterior graded response to Otx2 controls proliferation and differentiation of dopaminergic progenitors in the ventral mesencephalon. Development 135: 3459-3470.
PRAKASH, N., BRODSKI, C., NASERKE, T., PUELLES, E., GOGOI, R., HALL, A. PANHUYSEN, M., ECHEVARRIA, D.,-SUSSEL, L., WEISENHORN, D.M., MARTINEZ, S., ARENAS, E., SIMEONE, A. and WURST, W. (2006) A Wnt1regulated genetic network controls the identity and fate of midbrain-dopaminergic progenitors in vivo. Development 133: 89-98.

PRAKASH, N. and WURST, W. (2006). Development of dopaminergic neurons in the mammalian brain. Cel/ Mol Life Sci63: 187-206.

PUELLES, E., ACAMPORA, D., LACROIX, E., SIGNORE, M., ANNINO, A. TUORTO, F., FILOSA, S., CORTE, G., WURST, W., ANG, S. L. and SIMEONE A. (2003). Otx dose-dependent integrated control of antero-posterior and dorsoventral patterning of midbrain. Nat Neurosci 6: 453-460

PUELLES, E., ANNINO, A., TUORTO, F., USIELLO, A., ACAMPORA, D., CZERNY, T., BRODSKI, C., ANG, S. L., WURST, W. and SIMEONE, A. (2004). Otx2 regulates the extent, identity and fate of neuronal progenitor domains in the ventral midbrain. Development 131: 2037-2048.

ROFFLER-TARLOV, S., MARTIN, B., GRAYBIEL, A.M. and KAUER, J.S. (1996) Cell death in the midbrain of the murine mutation weaver. JNeurosi 16:1819 1826.

SCHEIN, J.C., HUNTER, D.D. and ROFFLER-TARLOV, S. (1998). Girk2 expression in the ventral midbrain, cerebellum, and olfactory bulb and its relationship to the murine mutation weaver. Dev Bio/204:432-450.

SIMEONE, A. (2005). Genetic control of dopaminergic neuron differentiation. Trends Neurosi28: 62-65

SMIDT, M.P. and BURBACH, J.P. (2007). How to make a mesodiencephalic dopaminergic neuron. Nat Rev Neurosci8: 21-32.

SMITS, S.M., BURBACH, J.P. and SMIDT, M.P. (2006). Developmental origin and fate of meso-diencephalic dopamine neurons. Prog Neurobio/78: 1-16.

SONNIER, L., LE PEN, G., HARTMANN, A., BIZOT, J.C., TROVERO, F., KREBS M.O. and PROCHIANTZ, A. (2007). Progressive loss of dopaminergic neurons in the ventral midbrain of adult mice heterozygote for Engrailed1. JNeurosci27: 1063-1071.

WALLÉN, A., ZETTERSTRÖM, R.H., SOLOMIN, L., ARVIDSSON, M., OLSON, L. and PERLMANN, T. (1999). Fate of mesencephalic AHD2-expressing dopamine progenitor cells in NURR1 mutant mice. Exp Cell Res 253: 737-746.

\section{Further Related Reading, published previously in the Int. J. Dev. Biol.}

See Special Issue Pattern Formation edited by Michael K. Richardson and Cheng-Ming Chuong at: http://www.ijdb.ehu.es/web/contents.php?vol=53\&issue=5-6

Isthmus organizer and regionalization of the mesencephalon and metencephalon Harukazu Nakamura and Yuji Watanabe Int. J. Dev. Biol. (2005) 49: 231-235

The isthmic organizer and brain regionalization S Martínez

Int. J. Dev. Biol. (2001) 45: 367-371

Otx2 and HNF3beta genetically interact in anterior patterning

O Jin, K Harpal, S L Ang and J Rossant

Int. J. Dev. Biol. (2001) 45: 357-365

The role of Otx2 in organizing the anterior patterning in mouse

A Simeone and D Acampora

Int. J. Dev. Biol. (2001) 45: 337-345

Genetic and epigenetic control of midbrain dopaminergic neuron development

C Perrone-Capano and U Di Porzio

Int. J. Dev. Biol. (2000) 44: 679-687

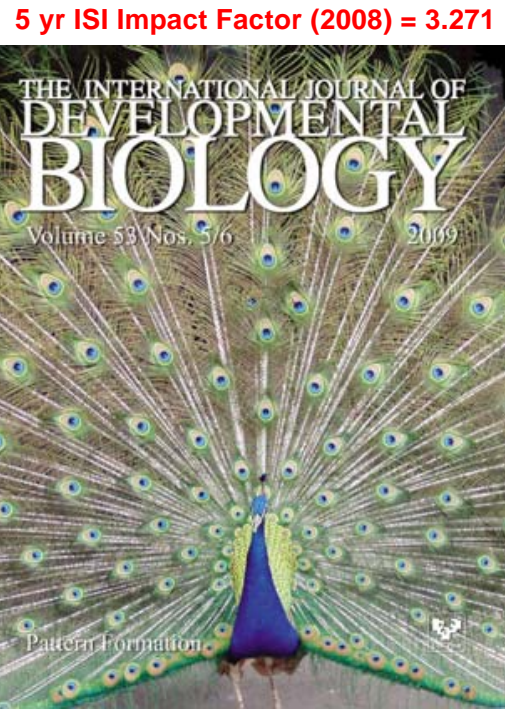

